\title{
Synote: Important Enhancements to Learning with Recorded Lectures
}

\author{
Mike Wald \\ ECS \\ University of Southampton \\ Southampton, UK \\ m.wald@soton.ac.uk
}

\author{
Yunjia Li \\ ECS \\ University of Southampton \\ Southampton, UK \\ yl2@ecs.soton.ac.uk
}

\begin{abstract}
This paper explains three new important enhancements to Synote, the freely available, award winning, open source, web based application that makes web hosted recordings easier to access, search, manage, and exploit for learners, teachers and other users. Synote uniquely achieves this through the creation of synchronized notes, bookmarks, tags, links, images and text captions, enabling users to easily find, or associate their notes or resources with, any part of a recording available on the web. Students surveyed would like to be able to access all their lectures through Synote. The facility to convert and import narrated PowerPoint PPTX files means that teachers can capture their lectures without requiring institution-wide expensive lecture capture systems. Crowdsourcing correction of speech recognition errors allows for sustainable captioning of the lecture while the development of an integrated mobile speech recognition application enables synchronized live verbal contributions from the class to also be captured through captions.
\end{abstract}

Keywords- speech recognition; recorded lectures; learning

\section{INTRODUCTION}

Commercial lecture capture systems (e.g. Panopto ${ }^{1}$, Echo360 ${ }^{2}$, Tegrity $^{3}$, Camtasia $^{4}$ ) can be expensive and do not easily facilitate educational student interactions. Synote ${ }^{5}$ overcomes the problem that while users can easily bookmark, search, link to, or tag the WHOLE of a recording available on the web they cannot easily find, or associate their notes or resources with, PART of that recording [1]. As an analogy, users would clearly find a text book difficult to use if it had no contents page, index or page numbers. Synote [2] can use speech recognition to synchronise audio or video recordings of lectures or pre-recorded teaching material with a transcript, slides and images and student or teacher created notes. Synote won the 2009 EUNIS International E-learning Award $^{67}$ and 2011 Times Higher Education Outstanding ICT Initiative of the Year award ${ }^{8}$. The system is unique as it is free to use, automatically or manually creates and synchronises transcriptions, allows teachers and students to create real time synchronised notes or tags and facilitates the capture and replay of recordings stored anywhere on the web in a wide range of media formats and browsers. Synote has been developed and evaluated with the involvement of users and with the support of JISC $^{9}$ and Net4Voice ${ }^{10}$. Figure 1 schematically illustrates the Synote system while figure 2 shows the Synote player interface. The technical aspects of the system, including the Grails Framework and the Hypermedia Model used, have been explained in detail elsewhere [3]. The synchronised bookmarks, containing notes, tags and links are called Synmarks (see figure 3). When the recording is replayed the currently spoken words are shown highlighted in the transcript. Selecting a Synmark, transcript word or Slide/Image moves the recording to the corresponding time.

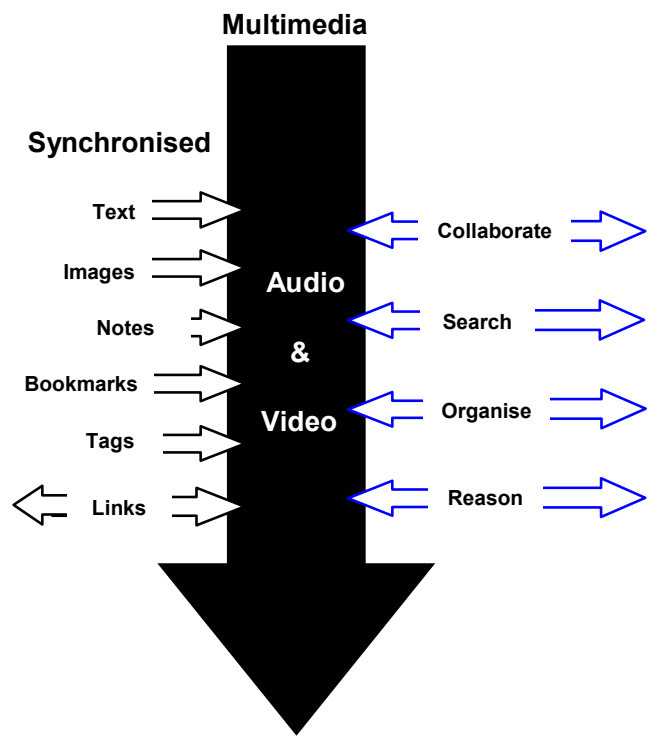

Figure 1. Synote overview

\footnotetext{
${ }^{1} \mathrm{http}: / /$ www.panopto.com/

${ }^{2} \mathrm{http}: / /$ echo360.com/

${ }^{3} \mathrm{http}: / /$ www.tegrity.com/

${ }^{4}$ http://techsmith.com/Camtasia

${ }^{5}$ www.synote.org

${ }^{6} \mathrm{http}: / /$ www.ecs.soton.ac.uk/about/news/2598

${ }^{7} \mathrm{http}: / /$ www.eunis.org/activities/tasks/doerup.html

${ }^{8} \mathrm{http}: / /$ www.ecs.soton.ac.uk/about/news/3874
}

\footnotetext{
${ }^{9} \mathrm{http}: / /$ www.jisc.ac.uk

${ }^{10} \mathrm{http}$ ://spazivirtuali.unibo.it/net4voice/default.aspx
} 


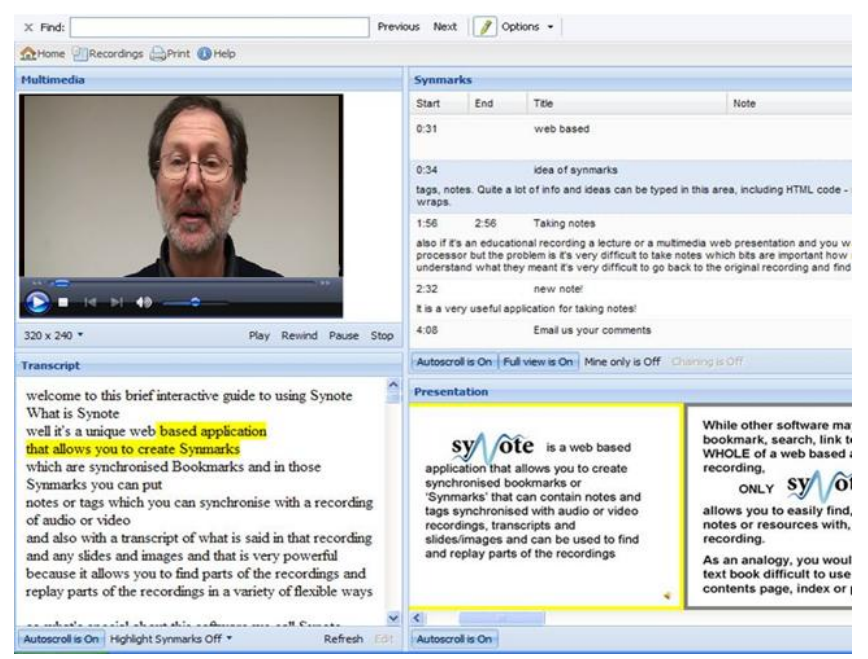

Figure 2. Synote Player Interface

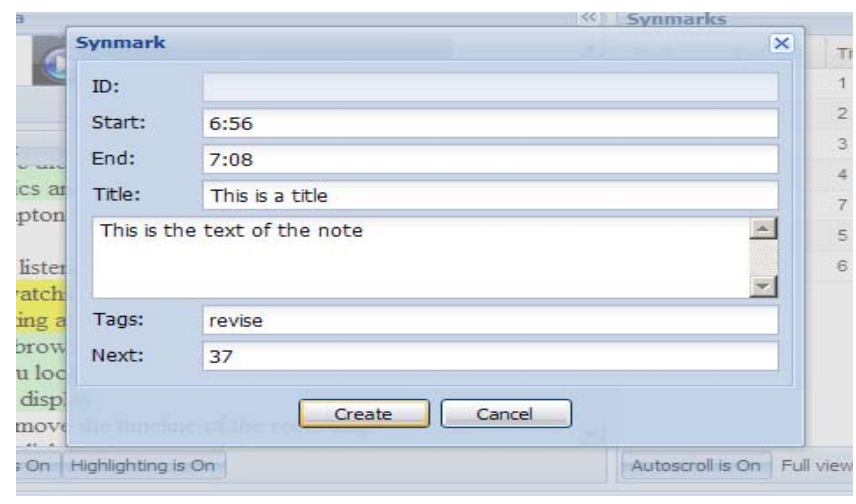

Figure 3. Synote Synmark Creation

The provision of text captions and images synchronized with audio and video enables all their communication qualities and strengths to be available as appropriate for different contexts, content, tasks, learning styles, learning preferences and learning differences.

Text can reduce the memory demands of spoken language; speech can better express subtle emotions; while images can communicate moods, relationships and complex information holistically. Synote's synchronised transcripts enable the recordings to be searched while also helping support non native speakers (e.g. international students) and deaf and hearing impaired students understand the spoken text. The use of text descriptions and annotations of video or images help blind or visually impaired students understand the visually presented information. So that students do not need to retype handwritten notes they had taken in class into Synote after the recording had been uploaded notes taken live in class on mobile phones or laptops using Twitter ${ }^{11}$ can be automatically uploaded into Synote. Until Microsoft Office 2010 was published with its undocumented changes to its saved .PPT format Synote could successfully create synchronized and searchable audio, transcripts and slides

\footnotetext{
${ }^{11} \mathrm{http}: / /$ twitter.com/synote

${ }^{12} \mathrm{http} / / /$ www.ecs.soton.ac.uk/about/news/2812
}

(including titles, text and notes) from narrated PowerPoint slides. The ability to import narrated PowerPoint files means that anybody can capture their lectures without requiring institution wide expensive lecture capture systems and section 2 of this paper reports on the development of a new PPTX to Synote xml format converter so that PowerPoint 2010 can be used successfully for recording lectures for Synote.

Synote builds on 12 years work on the use of speech recognition for learning in collaboration with IBM, and the international Liberated Learning Consortium [4] [5] and integration of the speaker independent IBM Hosted Transcription System with Synote has simplified the process of transcription giving word error rates of between $15 \%$ $30 \%$ for UK speakers using headset microphones. This compares well with the National Institutes of Standards (NIST) Speech Group reported WER of 28\% for individual head mounted microphones in lectures [6].

Commercial rates for manually transcribing and synchronising a lecture recording are typically around $£ 2 /$ minute $^{13}$ (rates vary dependent on quality and quantity). For automatic speech recognition to be used sustainably it must therefore cost less than this; including the manual correction of speech recognition transcription errors. A possible sustainable approach to obtaining accurate transcriptions is described in section 3 and involves students in the classes themselves correcting errors they find in the transcript, either voluntarily or through being paid or through being given academic credit.

The requirement of using headset microphones to obtain good speech recognition transcription accuracy means that contributions from students in the class are not easily recorded or transcribed. To address this problem Syntalk, a mobile transcription server, has been developed and is described in section 4. Section 5 summarises some evaluations that have been undertaken.

\section{POWERPOINT PPTX CONVERTER}

A narrated PowerPoint file can be used by a teacher to capture their lecture but each student would have to download the PowerPoint file to replay it on their own system. Office 2010 allows the narrated PowerPoint to be converted into a video that could be replayed by students but the video would not be captioned and the slide text would not be readable by a screen reader. The Synote PowerPoint converter enables the user to simply caption the recording using the slide notes and creates screen reader accessible text annotations for the slide images. The original Synote PPT converter was written in Java using the Apache POI9 library ${ }^{14}$ (POI-HSLF) which only provides an API for data extraction for Microsoft PowerPoint's original PPT format. There is no Java library available that supports Microsoft's 2010 PPT format file. The new Synote PPTX converter (Figure 4) changes the extension of the .PPTX file to .zip and extracts the slide text data and timing information and

\footnotetext{
${ }^{13} \mathrm{http}: / /$ www.automaticsync.com/caption/

${ }^{14} \mathrm{http}$ ://poi.apache.org/
} 


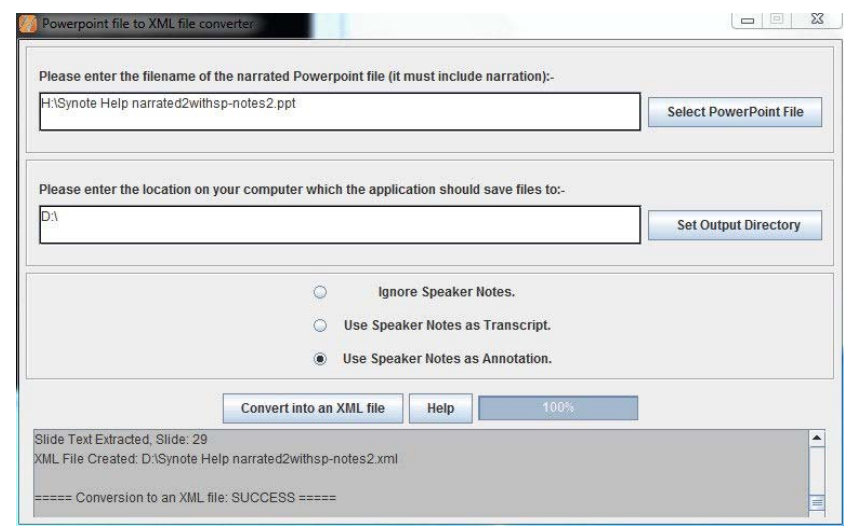

Figure 4. PowerPoint PPTX to Synote Converter

concatenates the audio .wav files that PowerPoint saves for each slide. The user needs to manually save the slide images as .png files. As PowerPoint does not record audio during slide transitions either the lecturer should not use any slide transitions or they should not speak during these transitions. The converter can automatically synchronise any text that the lecturer types into the slide notes with the narration either as a transcript or as an annotation.

\section{CROWDSOURCING SPEECH ReCOGNITION TRANSCRIPTION CORRECTION}

Universal Subtitles ${ }^{15}$ is a recent Mozilla Drumbeat project designed to allow users to manually caption web based videos but only allows one person at a time to create or edit the captions. YouTube and Synote both enable automatic speech recognition captioning of videos but also allowed only one person at a time to correct its captions. Since there is no correct version of the transcript in existence there is no way of knowing whether the person creating or correcting the captions is making errors or not. The approach that we have adopted therefore is to allow many people to edit the captions and then compare their edits. The newly developed crowdsourcing correction tool shown in Figure 5 stores all the edits of all the users and uses a configurable matching algorithm to compare users' edits to check if they are in agreement. The tool allows utterances from specified sections of the transcript to be presented for editing to particular users or for users to be given the freedom to correct any utterance. Administrator settings allow for different matching algorithms based on the closeness of a match and the number of users whose corrections must agree before the system accepts the edit as 'correct'. The red bar on the left of the utterance indicates to a user that they are not allowed to edit the utterance and the white on green tick on the right denotes that a successful match has already been achieved and so no further editing of the utterance is required. The green bar on the left of the utterance denotes that the required match for this utterance has yet to be achieved. Users can be awarded points for a matching edit

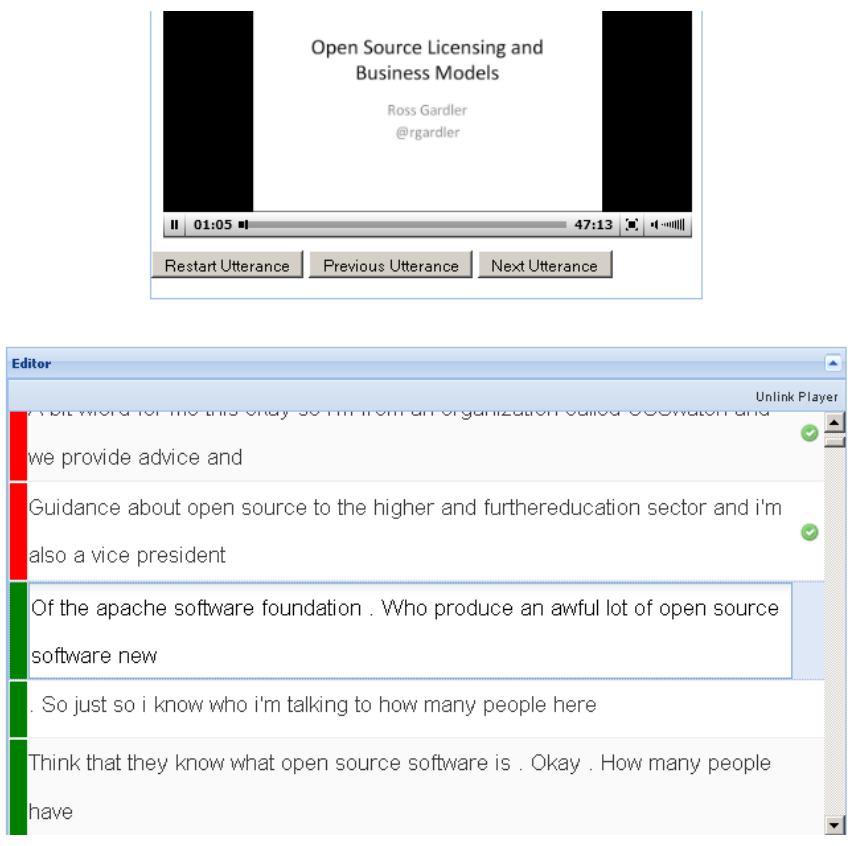

Figure 5. Crowdsourcing Correction Tool

and it is also possible to remove points for corrections that do not match other users' corrections.

\section{CAPTIONIng Contributions From StUdents USING SYNTALK}

Syntalk consists of two applications: an Android application (Figure 6) which is used by students to capture and transcribe and if required also correct their utterances and a web application (Figure 7) which is used by lecturers for managing the system. Users can choose to use any of three different free server based speech recognition systems, Google, EML ${ }^{16}$ or iSpeech ${ }^{17}$. At the start of a lecture the lecturer makes their lecture 'live' using the Syntalk web application control panel. Users can then select this live lecture on their Syntalk mobile application. When the user

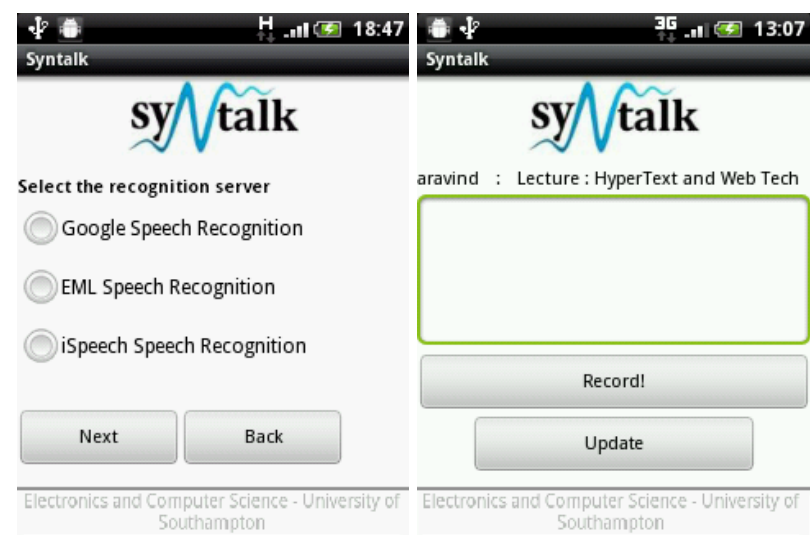

Figure 6. Syntalk Android Application

\footnotetext{
${ }^{15} \mathrm{http}: / /$ www.universalsubtitles.org
} 


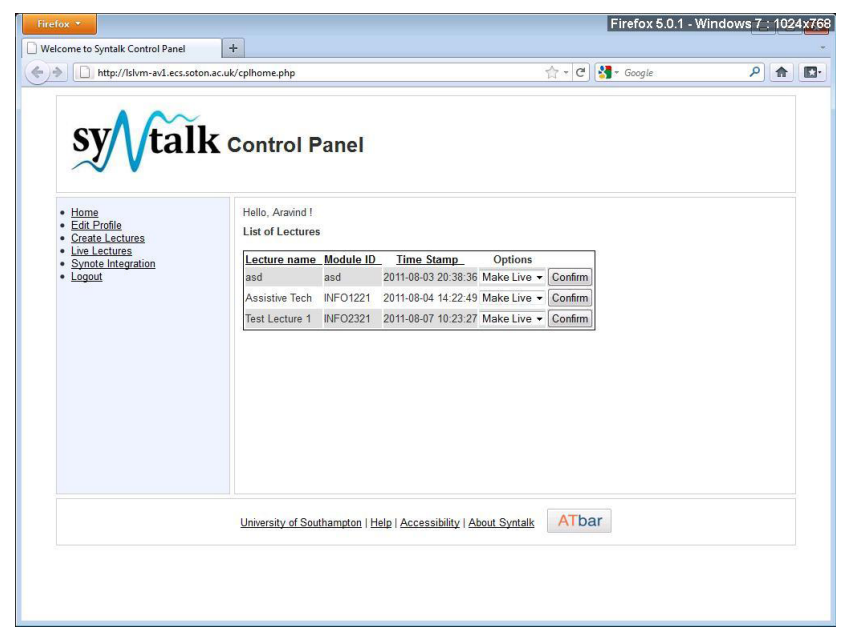

Figure 7. Syntalk Web Application

talks into their mobile's microphone the Syntalk mobile application sends the speech to the speech recognition server and when the transcribed text is returned by this server to the Syntalk application it is then sent to the Syntalk web server as well as being displayed on the mobile's screen for editing. If the user chooses to edit any speech recognition errors the corrected text is then also sent to the Syntalk server which creates an XML file containing the text captions and timings which can be uploaded into Synote as synchronized annotations. If everybody in a class used the Syntalk application on their personal mobile phone it would be possible to transcribe all spoken interactions. The current Syntalk application does not capture the spoken audio for Synote.

\section{Classroom Use and Evaluation of Synote}

Since 2008 Synote has been used by teachers in universities in the UK, Italy, Germany, Pakistan, Australia, US and Canada. Dr Wald has used Synote with over a hundred recordings of his lectures with synchronised transcripts and slides for his teaching of many hundreds of students on undergraduate and postgraduate Electronics and Computer Science (ECS) modules at The University of Southampton. At the time of writing there are over 1000 recordings publically available on Synote (most with synchronised transcripts) for students to use for their learning. The provision of a verbatim synchronised transcript enables students to concentrate on learning and take only brief synchronised notes in Synote (e.g. 'revise this section for exam', 'I don't understand this fully' etc.). This feature is of value to all students, not only deaf students who need to lipread or watch a sign language interpreter and so can't take notes or dyslexic students or non-native speakers who find it difficult to take notes. The fact that Synote is used and valued by all students means that non native speakers and disabled students feel more included and do not have to use special technology. Also the quality of recording from a teacher's wireless head worn microphone is significantly better than from small personal digital recorders placed by students at the front of the class. 5 ECS classes (two 1st year classes, one 3rd year class and two Masters classes) with approximately 200 students were surveyed, with 101 students filling in questionnaires about their experience with Synote. The questionnaire results showed that Synote's design to support a wide range of browsers was justified and appreciated with 54\% using Internet Explorer, 31\% using FireFox, 12\% using Google Chrome and 3\% using Safari. The results also confirmed that Synote was easy to use as over $80 \%$ of the respondents didn't need to read the Synote guide and rated Synote as 4 or 5 on a 5 point scale of ease of use with the remaining percentage rating it as 3 . The design of Synote to provide synchronised slides, video, audio and transcripts was also appreciated with over $80 \%$ respondents rating Synote as 4 or 5 for being useful overall as well as its slides, video and audio and transcript also each being useful with averages ratings of between 4.1 and 4.5. A slightly smaller percentage rated the Synmark option and the print out option as being useful with an average of 3.7 and 3.3 respectively on the 5 point scale used. One student commented that if Synote was used regularly then it would become second nature to use all these facilities. $97 \%$ of the students wanted all their lectures to be presented on Synote. 44 students in two of these 5 classes identified in more detail how using Synote affected their learning by indicating whether Synote improved or reduced or provided no change in the aspects of their learning shown in Table 1 which demonstrates that Synote improves most aspects for most students.

TABLE I. EFFECT OF SYNOTE ON LEARNING

\begin{tabular}{|c|c|c|c|}
\hline & improve & no change & reduce \\
\hline learning & $95 \%$ & $5 \%$ & $0 \%$ \\
\hline attention & $61 \%$ & $34 \%$ & $5 \%$ \\
\hline motivation & $50 \%$ & $50 \%$ & $0 \%$ \\
\hline efficiency & $77 \%$ & $23 \%$ & $0 \%$ \\
\hline enjoyment & $66 \%$ & $32 \%$ & $3 \%$ \\
\hline results & $69 \%$ & $31 \%$ & $0 \%$ \\
\hline notetaking & $53 \%$ & $30 \%$ & $18 \%$ \\
\hline attendance & $13 \%$ & $45 \%$ & $42 \%$ \\
\hline
\end{tabular}

A few students commented that some students' notetaking skills might not develop if all classes were recorded and presented on Synote. Some students commented that if they were ill or had serious problems they would normally still try and come in to classes because they were worried they would get behind in their work and would be unable to catch up, whereas with Synote they would be able to stay at home and get well secure in the knowledge that they would not miss anything as they could learn using the Synote recording. Students stated that it was important that they could depend on ALL lectures being recorded so they didn't find that the one lecture they missed hadn't been recorded. Of these 44 students $37 \%$ identified themselves as native English speakers, 26\% as fluent in English, 28\% as having good English language knowledge and $9 \%$ as having little English language knowledge. Non native speakers in 
particularly commented how valuable Synote was for them as note-taking in a foreign language was very difficult for them and it was sometimes difficult to understand lecturers' speech. One student commented that they could get words not understood in the transcript translated automatically by Google. Of these 44 students $7 \%$ identified themselves as having hearing disabilities, $2 \%$ visual disabilities, $11 \%$ learning disabilities and 7\% other disabilities.

Two overseas students wrote:

"Synote gives a very clear understanding of module ...It was very useful for me especially as I am a non English native speaker"

"I think Synote is a very good way to listen to lectures. If for example we miss the lecture we can actually listen to it in our own time or if we didn't really understand the lecture we can go back to it and listen to it carefully. I also like the highlighted part whenever the lecturer speaks on the text so we can't actually get lost within long texts."

Another student wrote:

"Synote is the best system I have ever seen for assistive technology, it is very useful for me to understand what the lecturer taught after class, I hope all school majors could integrate this system, thanks"

An evaluation of the Syntalk Android application conducted with 25 users having different levels of computer skills showed that the application was easy to use to capture and transcribe students' contributions. Users preferred Google speech recognition because of its recognition accuracy and transcription speed.

An evaluation of the Synote PPTX converter by a small group of students confirmed it was robust, simple and straightforward to use and efficiently converted $150 \mathrm{MB}$ PowerPoint files in less than 14 seconds.

Ten students tried the crowdsourcing editor and understood the material better as a result of the editing process suggesting that a marks incentive for editing might be justified on educational grounds. Some improvements were also suggested to the system which continues to be developed.

\section{CONCLUSION AND FUTURE WORK}

Commercial lecture capture systems are expensive and do not easily facilitate educational student interactions.
Synote has been shown to provide very well received enhancements to web based teaching and learning from recordings and to integrate well with other applications including PowerPoint, Twitter and Speech Recognition Software. The PPTX to Synote XML converter enables Lecturers to easily capture their lectures and replay them using Synote while Syntalk provides a simple and free way to also capture the rich student interactions that can occur in classrooms. Although Synote has much to offer even without synchronised lecture transcripts, there could be great educational benefits and a huge demand for speech recognition transcription if crowdsource editing makes it sufficiently accurate and affordable. A demonstration and further results of the use of the systems described in this paper will be presented at ICALT 2012.

\section{ACKNOWLEDGMENT}

The following ECS students were involved with the development of the technologies described in this paper: Kejun Huang, Chi Lok Koo, Kexin Liu, Raymond Mo, (PPTX to Synote XML Converter); Dawid Michal Koprowski, Karolina Kaniewska, Michael Kanani, Stella Sharma (Crowdsource Editing of Speech Recognition Captioning System for Synote); Aravind Vaithianathan (Syntalk Student Contribution Captioning System For Synote).

\section{REFERENCES}

[1] Whittaker, S., Hyland, P., Wiley, M. Filochat handwritten notes provide access to recorded conversations, Proceedings of CHI 1994 271-277.

[2] Wald, M., Wills, G., Millard, D., Gilbert, L., Khoja, S., Kajaba, J. and Li, Y. Synchronised Annotation of Multimedia. 2009 Ninth IEEE International Conference on Advanced Learning Technologies. 2009 594-596.

[3] Li, Y., Wald, M., Wills, G., Khoja, S., Millard, D., Kajaba, J., Singh, P. and Gilbert, L. (2011) Synote: development of a Web-based tool for synchronized annotations. New Review of Hypermedia and Multimedia. pp. 1-18.

[4] Leitch, D., MacMillan, T. Liberated Learning Initiative Innovative Technology and Inclusion: Current Issues and Future Directions for Liberated Learning Research. Saint Mary's University, Nova Scotia. 2003 http://www.liberatedlearning.com/

[5] Wald, M. and Bain, K. Enhancing the Usability of Real-Time Speech Recognition Captioning through Personalised Displays and RealTime Multiple Speaker Editing and Annotation. In Proceedings of HCI International 2007: 12th International Conference on HumanComputer Interaction, Beijing pp 446-452.

[6] Fiscus, J., Radde, N., Garofolo, J., Le, A., Ajot, J., Laprun, C., (2005) The Rich Transcription 2005 Spring Meeting Recognition Evaluation, National Institute Of Standards and Technology 